\title{
Research Progress of Intelligent Image Recognition
}

\author{
Kai $\mathrm{Wu}^{1, \text { a }}$ \\ ${ }^{1}$ School of Computer Science and Engineering, Nanjing University of Science and \\ Technology, Nanjing 210094, China \\ a1339617668@qq.com
}

\begin{abstract}
Intelligent image recognition is a technique that enables image information to be digitized quickly and accurately. Optical Character Recognition (OCR) is an important technology in the field of application research such as machine vision and pattern recognition. This paper summarizes the background and research progress of intelligent image recognition, analyzes the present situation and technical characteristics of intelligent image recognition, and try to figure out how the intelligent image recognition system is operated. Finally, we reported the intelligent image recognition algorithm, such as Hu's moment invariants and DS evidence reasoning. From the concept principle to the algorithm, the characteristics of intelligent image recognition technology are analyzed in detail. Hope to be fully aware of the basis of it, to find ways to improve the optimization and in the end to provide more convenience for people under the information age.
\end{abstract}

Keywords: intelligence, image recognition, OCR, image process

\section{Introduction}

With the development of information technology, handheld terminal equipment has gradually become the main tool for personal information processing. At the same time, with the popularity of the Internet and enhancement of the function of these devices towards the small, intelligent direction, the relevant efficient information technology software and hardware products will emerge as the requirement. In this era of rapid growth of information, more and more data is stored in electronic form and can be transmitted over the Internet, in the form of character flow it can walk in every corner of the world. At the same time, the traditional information products such as paper and other materials as a medium, because of its official nature and low-requirement, are widely used in many occasions. But because of its high cost, small-storage capacity, inconvenient, difficult to sort and other factors, they bring a lot of inconvenience for people's lives. Thus, the use of existing equipment to transform paper and other media information automatically and quickly into electronic ones on the digital devices, playing the advantages of electronic products is an urgent need to solve the problem. And it will accelerate text information storing speed, easily organize and store information, improve social productivity.

At present, the text input of the paper text mainly relies on manual operation, including computer keyboard input, tablet input, voice input, etc. Although the operation is simple, its speed of conversion to electronic information is not high, for a large number of paper documents. Using these methods will cost a lot of manpower and time resources. Therefore, how to make the image information can be quickly and accurately digitized into a valuable application is an urgent need. OCR, that is, Optical Character Recognition theory [1] is proposed to solve this problem effectively, it refers to the optical character recognition technology, machine vision and pattern recognition and other applications. 
This technology can transform the text information obtained from the document and other materials by using the optical imaging into the image file of black and white lattice form. It could use various types of pattern recognition algorithm to analyze the morphological characteristics of characters, and determine the identification of output character text, and then transform the text in the text format, or other types of paper information into digitized ones. OCR technology can digitize the image content, so that people can process the paper materials with low cost, fast and high quality storage, the technology will lead the convenient digital processing technology into the traditional media, to achieve the development and utilization of resources and facilitate the people in low-cost learning work. The rapid development of computer technology has important significance in the development and change of the whole society. Ant it also has an important positive role in promoting the progress of the whole social level [2].

\section{Research Status of Intelligent Image Recognition System}

The concept of OCR was first proposed by the German scientist Tausheck in 1929, after which scholars have made further research on this area. American scientist Handel first realized using OCR theory to identify the text. After 30 years of continuous development and improvement, IBM successfully made the world's first generation of OCR products. But it can only be used into the application of simple digital identification, such as: mail on the postal code recognition. In the 1970s, Japanese scholars began to study the use of OCR technology for Chinese character recognition applications, and did a lot of work. Hitachi and Fujitsu companies have also developed their own OCR products. In recent years, with the enhancement of hardware equipment into technology, the speed up of operation and other factors, ORC technology has also been further development. Foreign information departments, press and publishing units have the relevant OCR software, such as: The United States ABBYY FineReader pro v10 and Readiris Pro asia v12. The software is highly intelligent, with good tilt correction function, layout analysis function, error correction and higher recognition rate. They are basically improving the preparation of the traditional character recognition technology, and its technology mainly is template matching method, statistical method, wavelet transform $[3,4]$.

\section{Overview of Intelligent Image Recognition Technology}

\subsection{The Principle of Intelligent Image Recognition Technology}

The computer intelligent image recognition technology is, under the support of computer technology and information technology, through the computer system to deal with and analyze the input image, in order to achieve accurate identification of different models of objects, which is shown in Figure 1.

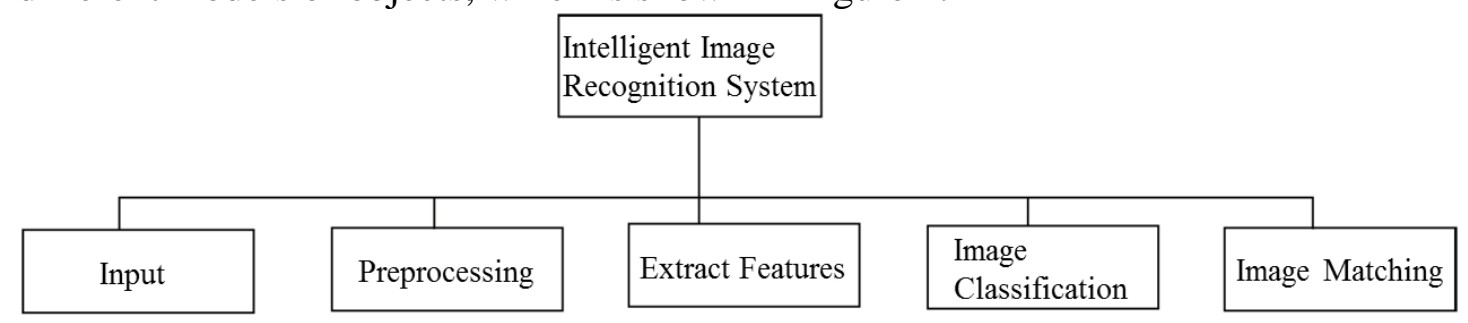

Fig. 1 The diagram of intelligent image recognition system

In order to help people to get better information from the image, the computer intelligent image recognition technology can effectively use different spatial mapping 
to image recognition, and then fully realize the image class and the characteristics, accurately comprising and matching, to ensure the clarity of image processing, which aim to improve the ability of identifying the system's image [5].

\subsection{The Properties of Intelligent Image Recognition Technology}

Computer intelligent image recognition technology has three major characteristics: 1) Large amount of information. Under meeting the conditions of the computer configuration, running system, storage capacity, image information processing methods, on the basis of two-dimensional information need large amount of information, image recognition process requires sufficient preparation conditions; 2) Strong relevance. In the computer system, there is a strong correlation between the pixels of the image, so the image information should be effectively compressed in the process of intelligent image recognition to achieve the accurate classification and matching of the image information. 3) Computer intelligent image recognition is mainly evaluated by the people, so the human factors have a great deal of constraints on the image observation and evaluation, which is easy to affect the quality of image recognition [6].

\subsection{Overall Design of Embedded Intelligent Image Processing System}

Image recognition and tracking algorithm is complex and computationally large, the system real-time and reliability are very high requirements, for which the use of TI's high-end DSP chip TMS320C6455 as the processing core of the system. At the same time with Altera's Cyclone III series Programming logic chip, which mainly completes the timing control, image preprocessing and image overlay and other auxiliary functions, thus greatly improving the system scalability and flexibility [7]. The overall structure of the embedded intelligent image processing system is shown in Figure 2.

The embedded intelligent image processing system is composed of video signal conversion module, image data processing module and data communication module. The main functions of each module are as follows:

Video signal conversion module: it is to achieve video encoding and video decoding capabilities, while the completion of a variety of video signal collection and conversion.

Image data processing module: the core of the entire system module, composed of DSP and FPGA, FPGA is to complete the real-time video data acquisition, analysis and processing, DSP computing resources are used as much as possible for algorithm processing.

Data communication module: the completion of image data transmission and multi-chip communication, by the development of Gigabit Ethernet data transmission interface and high-speed serial interface components. 


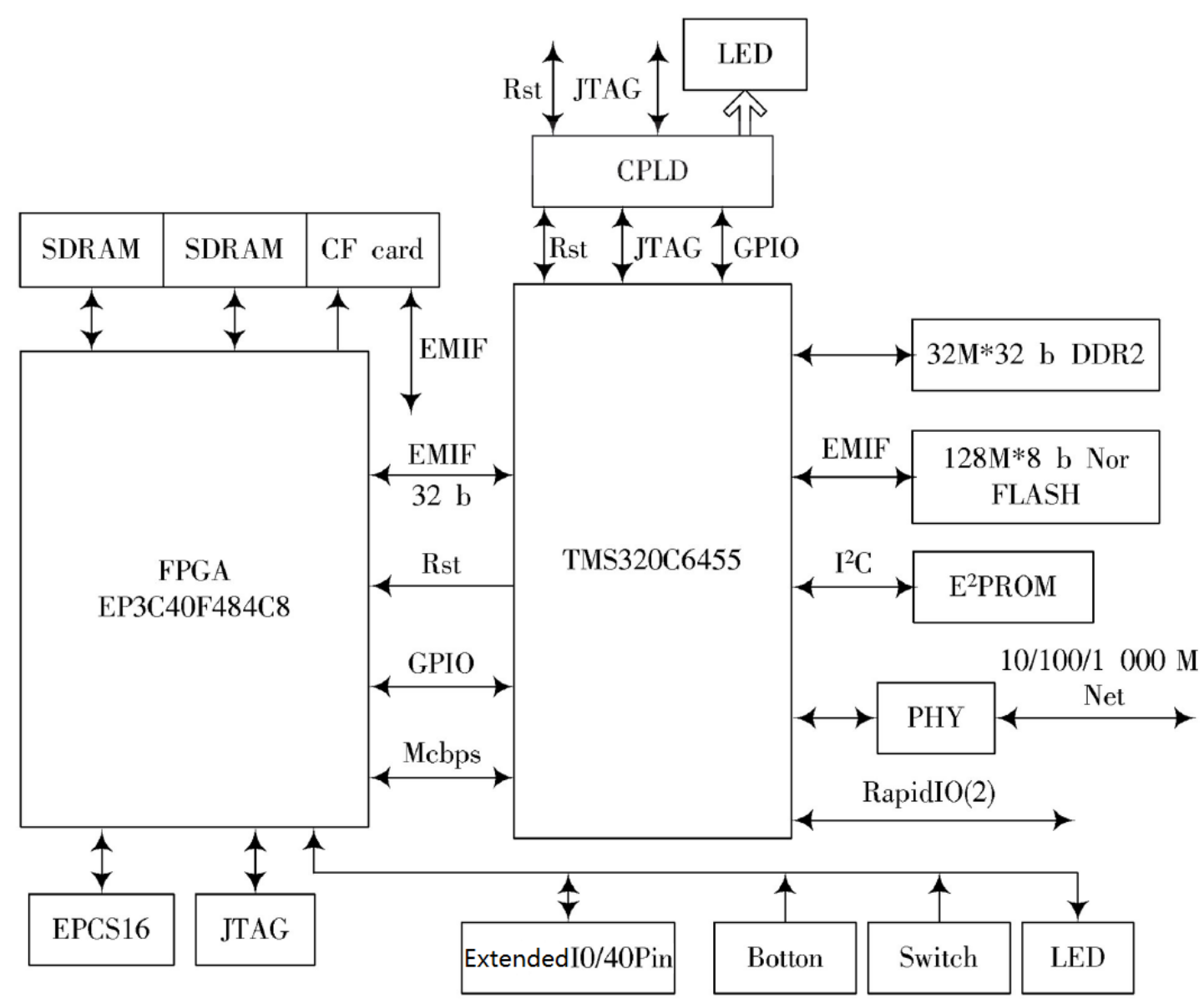

Fig. 2 The diagram of image processing system

\section{Realization of Intelligent Image Recognition Technology}

Intelligent image recognition system is divided mainly into three categories [8]:

(1) template matching method: according to the known pattern to another map to find the appropriate mode of the method.

(2) statistical identification method: it is known as decision-making theory. It extracts the features from the patterns represented by the eigenvectors, and identifies the patterns by dividing the feature space, which is based on the decision function.

(3) Syntactic analysis method: it is based on the knowledge of language grammar, especially in the form of formal theory developed in the form, each model is composed of sub-mode or model elements of the composition. It points out the similarity between the structure of the pattern and the syntax of the language, and analyzes the pattern structure according to a given set of grammatical rules to achieve the pattern recognition.

\section{Research on the Algorithm of Intelligent Image Recognition Technology}

\subsection{Hu moment invariants}

The invariant moment theory has the invariant mathematical features in the movement and change, and then proposes the invariant moment method to carry on the image recognition algorithm.

$$
\eta_{p q}=\sum_{m=1}^{M} \sum_{n=1}^{N}(\mathrm{~m}=\bar{x})^{p}(\mathrm{n}-\bar{y})^{q} f(m, n)
$$


The second and third order central moments are constructed to form discrete invariants of seven different expressions.

\subsection{D-S Evidence Reasoning}

If the subset of $\Theta$ is $A$, and $\mathrm{m}(A)>0$, then $A$ is the focus element of the evidence (referred to as the focal element), and the "kernel" is the general set of all the coke elements. The evidence body of several $(A, \mathrm{~m}(A))$ constitutes the evidence, and the three measure functions on $R$ can be defined by the evidence body, as follows:

$$
\begin{aligned}
& \operatorname{Bel}(A)=\sum\{m(B) \mid B \subseteq A, B \neq \varnothing\} \\
& P l(A)=\sum\{m(B) \mid A \cap B \neq \varnothing\} \\
& q(A)=\sum\{m(B) \mid A \subseteq B \subseteq \Theta\}
\end{aligned}
$$

Using the Dempster combination rule, two or more confidence functions can be obtained, based on the calculation it can be derived from different sources of confidence in the orthogonal sum, and then find a new confidence function [9].

\section{Conclusion}

The development of science and technology has promoted the intelligentization of the Internet age. In the new field of computer artificial intelligence, image recognition is one of the main research contents, and has achieved remarkable achievements. At present, computer intelligent image recognition algorithms and techniques have been widely used in various fields. In this paper, we have better understanding of their research progress, technical characteristics, implementation methods and corresponding algorithms. Although it has some shortcomings in present application process and still need to be explored, it always keeps making progress to provide better support for social development.

\section{Reference}

[1] X.Q. Ding, The problem of Chinese character recognition [J]. Journal of Electronics, 2002, 30: 1364-1368.

[2] Q. Wu, L. Liu, Research on auto-recognition technology of automobile license [J]. China Transportation Information Industry, 2003, 5: 88-90.

[3] Y.L. Yu, Digital Image processing and pattern recognition [M]. South China University of Technology Press, 1990

[4] W. Niblack. An introduction to digital image processing [J]. Prentice Hall. 1986: 115-116.

[5] M.K. Zhang, Discussion on computer intelligent image recognition technology [J]. Technology Information, 2009.

[6] W. Zhou, A brief introduction to the application of computer identification technology [J]. Technology Information, 2010.

[7] WILSON A. Camera link standard advances interfacing [J]. Vision systems design, 2015, 8: 43-44.

[8] H.M. Luo, Z. Peng, Z.G. Li, A remote video surveillance system based on intelligent image recognition [J]. Computing technology and automation, 2005, 24: 51-53

[9] H.B. Du, C.Y. Liu, Image recognition algorithm based on convex template for target morphology [J]. Computer and Network, 2011, 14: 49-52. 